\title{
Escenarios de subida del nivel medio del mar en los mareógrafos de las costas peninsulares de España en el año 2100
}

\section{Sea level rise scenarios in the peninsular Spanish tide gauges in the year 2100}

\author{
Pablo Fraile Jurado y Miguel Fernández Díaz ${ }^{1}$
}

\section{INTRODUCCIÓN}

La subida del nivel medio del mar es la consecuencia directa del actual cambio climático sobre la cual ha existido un mayor consenso tanto en los sucesivos informes del IPCC (Bruce et al., 1996; IPCC, 2007; IPCC, 2013) como en otros informes internacionales (Schubert et al., 2006) en cuanto a su ocurrencia.

Desde que se instalaron los primeros mareógrafos en el planeta, a finales del siglo XIX, se ha observado una subida media del nivel del mar cuantificada en $+1.7 \mathrm{~mm} /$ año en el periodo 1901-2010 (IPCC, 2013). Asimismo, se ha identificado una aceleración en este proceso, publicándose tendencias de +2.0 mm año desde 1971 a 2010, y de $+3,1 \mathrm{~mm} /$ año para el periodo 1993-2010 (IPCC, 2013). Estas tendencias de cambio del nivel del mar son coherentes tanto en registros de mareógrafos, única fuente de información hasta 1992, como en los datos procedentes de los satélites altimétricos, que desde 1992 miden la altura de la superficie marina en aguas oceánicas.

\footnotetext{
${ }^{1}$ Pablo Fraile pertenece a la Universidad de Sevilla, pfraile@us.es

Miguel Fernández es miembro del Grupo de Ordenación del Litoral y Tecnologías de Información Territorial, dirigido por José Ojeda Zújar. migfedi@gmail.com
} 
La mayor parte de la discusión científica sobre esta cuestión se ha centrado en la estimación global de la subida futura, generalmente empleando el horizonte del año 2100. Si bien se ha podido constatar un descenso de las predicciones de subida desde los modelos elaborados durante los años 80 hasta el presente, la comunidad científica participa en la actualidad en una discusión entre los modelos del IPCC y los de una serie de autores (Rahmstorff, 2007; Pfeffer et al., 2008) que han estimado subidas muy superiores a las del IPCC basándose en la observación empírica de los registros recientes de mareógrafos y satélites altimétricos. Los resultados de estos trabajos demuestran que el nivel del mar ha subido a un ritmo superior al previsto por los modelos del IPCC desde el año 1990, fecha que diferentes informes (IPCC, 2001; IPCC, 2007) tomaban como referencia para pronosticar el comportamiento futuro del nivel del mar, por lo que han corregido al alza las previsiones del IPCC.

Más allá de la discusión científica inherente a los registros, análisis y cálculo de expectativas de cualquier variable ambiental, la cuestión sobre la magnitud de la previsible subida del nivel medio del mar tiene una gran importancia para otro tipo de estudios. La magnitud de la futura subida del nivel medio del mar es un parámetro de entrada crítico para análisis sobre erosión potencial (Bosello et al., 2012), sobre inundaciones esperadas en áreas urbanas (Nichols y Cazenave, 2010; Curtis y Schneider, 2011), naturales (Katsman et al., 2011; Fraile y Ojeda, 2013) o en infraestructuras (Hallegate et al., 2011), o sobre pérdida de calidad de aguas subterráneas (Loacigia et al., 2012; Nicholls, 2011).

Del mismo modo, y aunque esté ausente en la mayoría de las leyes urbanísticas y de planificación territorial de ciudades y territorios costeros, el uso de un umbral que delimite los territorios que podrían verse inundados en el futuro es esencial para realizar una planificación que considere todos los riesgos naturales, especialmente los costeros. Algunos países ya han aplicado este tipo de umbrales a sus normativas urbanísticas, como es el caso de EEUU (Karl et al., 2009; Titus y Anderson, 2009) o de Alemania, que aplica la posible subida del nivel del mar publicada por Rahmstorf como umbral para delimitar los territorios en los que se puede construir o no (Schubert et al., 2006).

La evidente importancia de esta variable no suele conllevar un tratamiento detallado al realizar estudios que requieren un planteamiento local. Desde este punto de vista, los cambios observados en el nivel medio del mar de una costa son necesariamente relativos (Lisitzin, 1974), y presentan dos componentes: i) una global (eustasia), dependiente de la masa y el volumen del agua presente en las cuencas oceánicas ( $y$ variable por tanto por distintos fenómenos como el calentamiento global), y ii) otra local, explicada tanto por los movi- 
mientos verticales de la superficie emergida (causados por tectónica local o regional, compresión de sedimentos...) como de la superficie marina situada frente a la costa.

Al estar anclados a la superficie emergida, los registros de los mareógrafos son una excelente fuente de información para evaluar la magnitud de los cambios relativos del nivel medio del mar, puesto que engloban ambas componentes. De cara a estimar las expectativas futuras de cambio del nivel medio del mar a escala local, es preciso combinar adecuadamente tanto la información procedente de los registros de los mareógrafos (medidas frecuentemente en tendencias de cambio anual) como de los escenarios globales de cambio del nivel medio del mar, elaborados a partir de complejos modelos climáticos.

La aproximación más precisa realizada sobre esta cuestión se abordó por Titus y Narayanan (1998), que proponían eliminar la componente global existente en el cálculo de una tendencia pasada en una serie temporal de un mareógrafo, aislando el término local. Dado que asumían que el término local debe ser estable en el tiempo (al estar asociado frecuentemente a fenómenos tectónicos con escasa variabilidad interanual), su metodología permitía sumar la componente local a las expectativas de un modelo global de cambio del nivel medio del mar. No obstante, esta propuesta exigía el uso de de series temporales de más de 100 años de duración, por lo que su repercusión ha sido escasa, dado que no es común disponer de este tipo de datos. Aun así, este trabajo ha recibido numerosas citas (Nicholls et al., 2011; Emrich y Cutter, 2011; Moss, 2011), aunque la mayoría de ellas referidas a la introducción del concepto de probabilidad que a la idea de aislar el término local en las tendencias observadas.

El IPCC (2007) ha alentado la elaboración de estudios locales para obtener datos fiables sobre el comportamiento pasado y futuro del nivel medio del mar, de cara a una planificación territorial más efectiva. No obstante, son muy frecuentes los análisis que únicamente consideran la variabilidad futura del nivel del mar como el resultado de un fenómeno exclusivamente global, homogéneo en su magnitud en las costas de todo el planeta.

En España es poco frecuente la publicación de este tipo de trabajos. Desde las instituciones responsables de las redes de mareógrafos se han calculado las tendencias de cambio del nivel medio del mar para cada mareógrafo. Sin embargo, estos estudios no se han plasmado en otros trabajos derivados en los que se evalúen niveles del mar futuros a partir de los registros locales. En este sentido, los principales trabajos de referencia sobre cambio climático en España emplean un horizonte fijo de subida del nivel medio del mar de 1 metro para todas las costas españolas para finales del siglo XXI (Méndez et al., 2004; 
Moreno, 2006), a excepción de trabajos de carácter local en diferentes emplazamientos como el litoral valenciano, (Pardo Pascual, 1989), gaditano (Fraile y Ojeda, 2012) o canario (Fraile et al., 2015).

El objetivo del trabajo es realizar una evaluación de las expectativas de subida del nivel medio del mar en los mareógrafos situados en la costa peninsular española en el año 2100, a partir del análisis combinado de las series temporales de nivel del mar registradas en los mareógrafos y de los modelos globales de cambio futuro del nivel medio del mar.

DATOS

Para la elaboración de este trabajo se han empleado dos tipos de series temporales: i) las registradas en los mareógrafos de la costa peninsular española, y ii) la elaborada por el Commonwealth Scientific and Industrial Research Organisation (CSIRO), de carácter global, a partir de las series más largas de mareógrafos del planeta, entre las que no se encuentra ninguna de las analizadas en este trabajo.

Los registros de las series de los mareógrafos peninsulares proceden del Permanent Service for Mean Sea Level (PSMSL), organismo internacional que recibe y publica las series temporales de los mareógrafos de diferentes instituciones nacionales. El formato de los registros de niveles del mar empleado por este organismo es el RLR (Revised Local Reference). Este formato, expresado en $\mathrm{mm}$, reduce a todos los mareógrafos del mundo a un datum común sobre su propio cero hidrográfico (valor más bajo posible), al que se le añaden arbitrariamente $7000 \mathrm{~mm}$, con la intención de evitar la presencia de registros negativos. En el caso español, los mareógrafos proceden de tres instituciones: el Instituto Español de Oceanografía (IEO), Puertos del Estado (PE) y el Instituto Geográfico Nacional (IGN) (tabla 1). La red española de mareógrafos se caracteriza por la escasez de series temporales largas. Tan solo los mareógrafos del IEO situados en Vigo, A Coruña, Santander, Málaga y Cádiz presentan series completas (o con escasas lagunas) de más de 30 años (figura 1). La mayor parte del resto de la red se puso en marcha en la década de los 90 (figura 2). Adicionalmente, la distribución espacial de los mareógrafos peninsulares dista mucho de ser homogénea (figura 3). 
TABLA 1

MAREÓGRAFOS SITUADOS EN LAS COSTAS IBÉRICAS ESPAÑOLAS, E INSTITUCIONES QUE LOS GESTIONAN.

\begin{tabular}{ll}
\hline Mareógrafo & Institución \\
\hline Huelva & Puertos del Estado \\
Bonanza & Puertos del Estado \\
Cádiz & Instituto Español de Oceanografía \\
Algeciras & Instituto Español de Oceanografía \\
Málaga & Puertos del Estado \\
Almería & Instituto Geográfico Nacional \\
Cartagena & Instituto Español de Oceanografía \\
Alicante & Instituto Español de Oceanografía \\
Valencia & Puertos del Estado \\
Barcelona & Puertos del Estado \\
Bilbao & Puertos del Estado \\
Santander & Instituto Español de Oceanografía \\
Gijón & Puertos del Estado \\
A Coruña & Instituto Español de Oceanografía \\
Vigo & Instituto Español de Oceanografía \\
\hline
\end{tabular}

FIGURA 1

SERIES TEMPORALES DE NIVEL MEDIO ANUAL DEL MAR, ANALIZADAS EN LOS MAREÓGRAFOS CON SERIES LARGAS DE REGISTROS

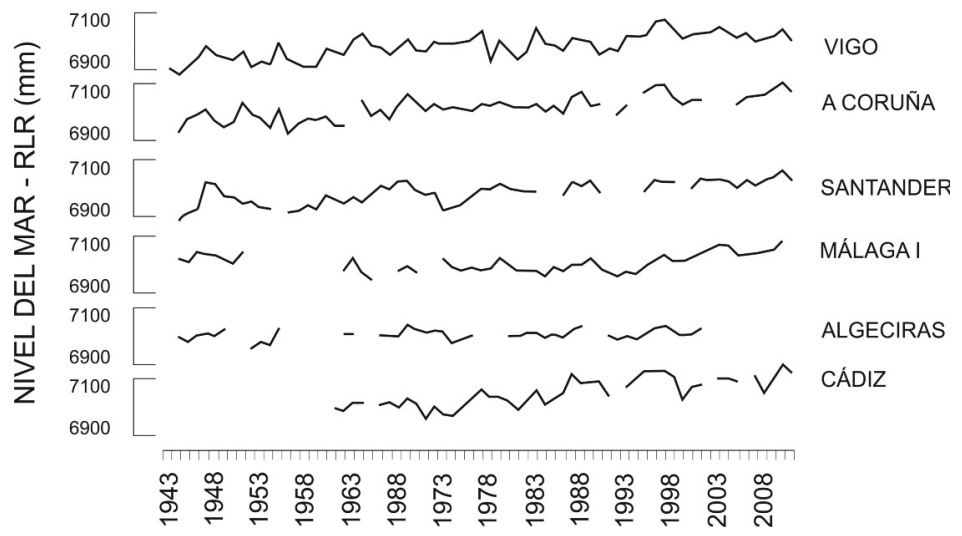


FIGURA 2

SERIES TEMPORALES DE NIVEL MEDIO ANUAL DEL MAR, ANALIZADAS EN LOS MAREÓGRAFOS CON SERIES CORTAS DE REGISTROS

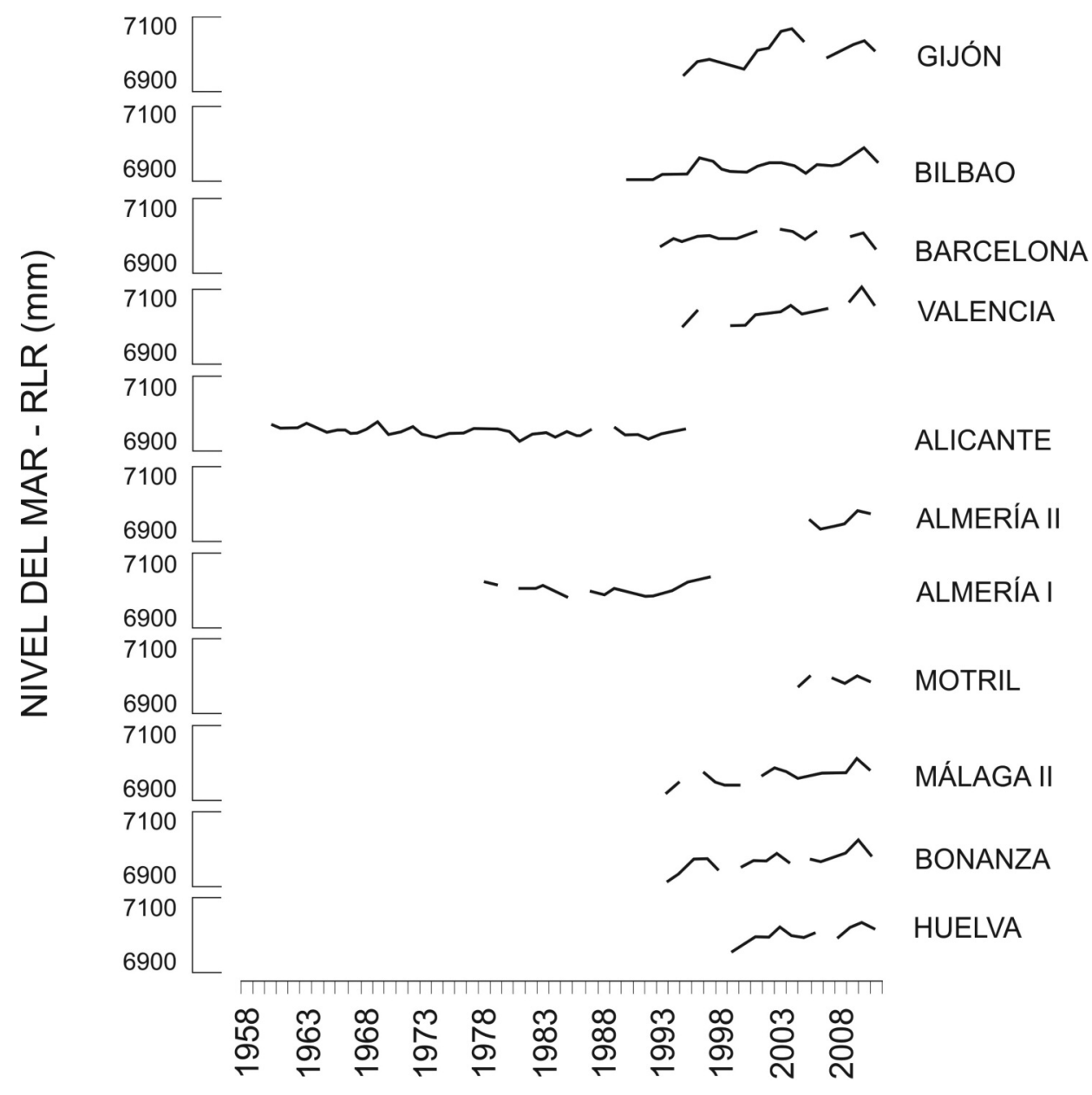




\section{FIGURA 3}

\section{MAPA DE LA DISTRIBUCIÓN DE LOS MAREÓGRAFOS LOCALIZADOS EN LAS} COSTAS PENINSULARES ESPAÑOLAS

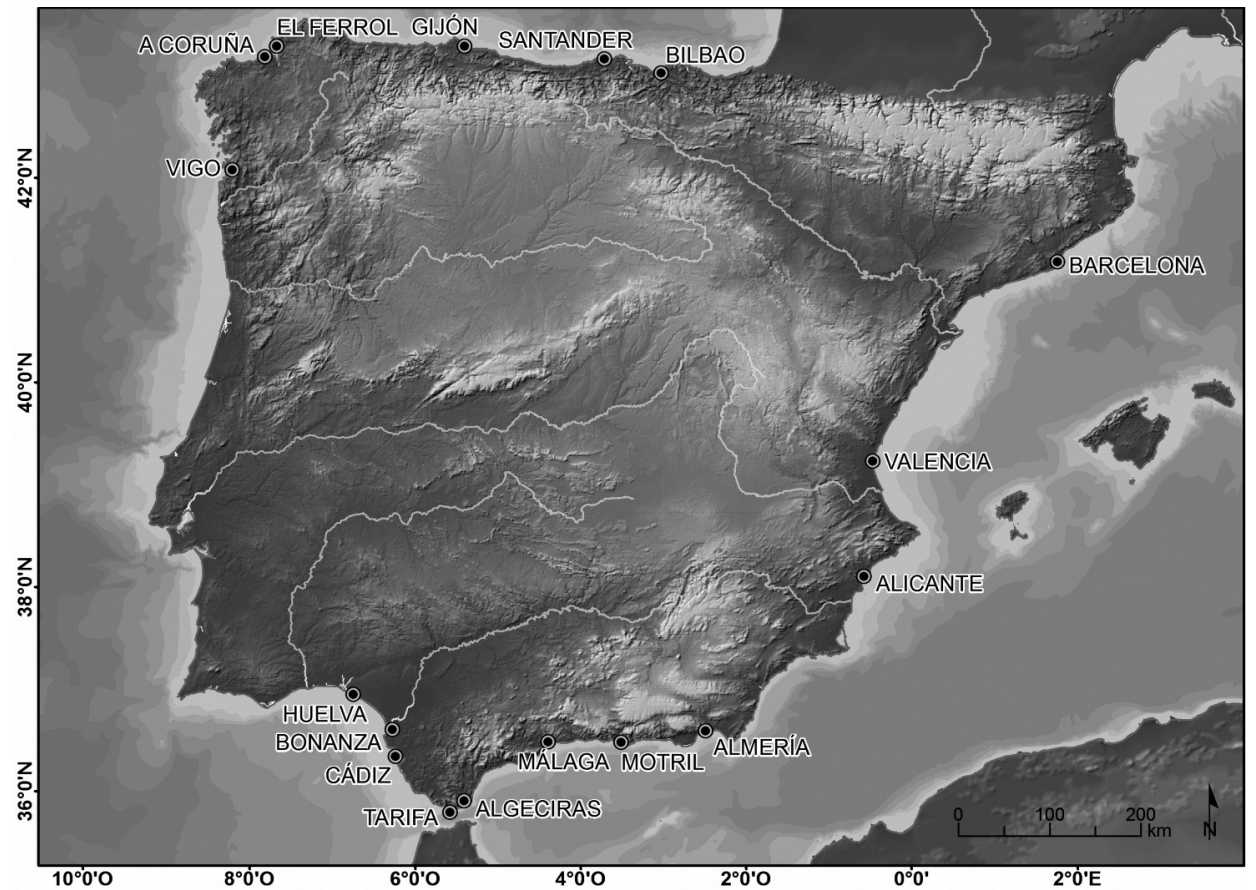

Aunque algunos de los mareógrafos analizados tienen registros para series temporales más largas de las que se han empleado en este trabajo (Cádiz, Alicante o Tarifa), se ha descartado su uso debido a la aparente falta de fiabilidad identificada en trabajos previos de los registros que comienzan en 1940 (Fraile, 2011). Igualmente, la serie temporal del mareógrafo de Gibraltar fue desestimada de inicio por anomalías en los datos reportadas en la propia web del PSMSL.

La serie de niveles del mar globales utilizada para este trabajo fue publicada por el CSIRO (Church y White, 2011), y proporciona un valor único de nivel medio del mar para cada año desde principios del siglo xx (figura 4). Se ha elaborado a partir de la ponderación de los registros de los mareógrafos de todo el planeta. 
FIGURA 4

SERIE TEMPORAL DEL NIVEL MEDIO DEL MAR GLOBAL PARA EL PERÍODO 1909-2011

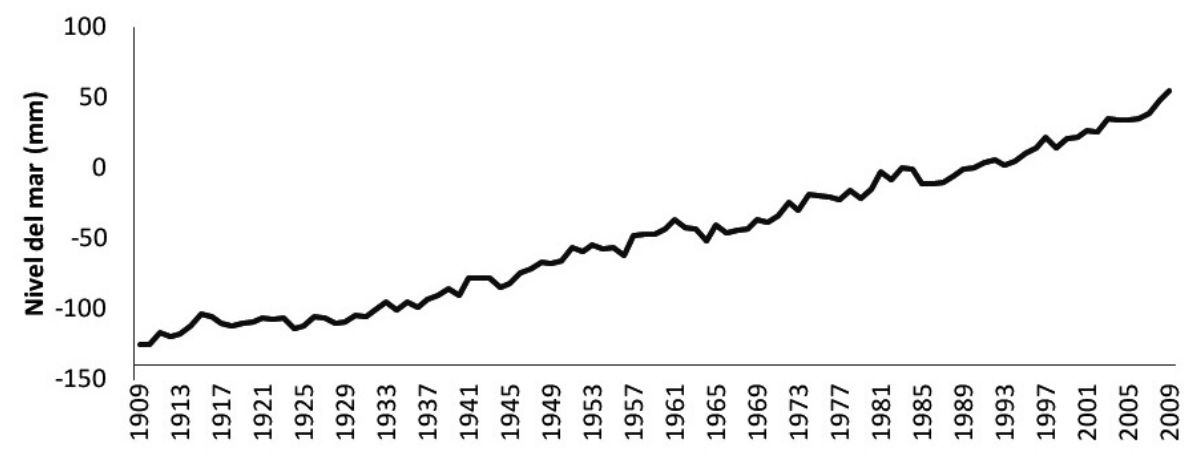

\section{MÉTOdOS}

La metodología presentada en este trabajo se ha diseñado con el propósito de calcular futuros niveles del mar combinando las tendencias observadas hasta el presente con la subida esperada al final del siglo XxI de acuerdo con diferentes escenarios.Para ello, los pasos seguidos en el cálculo de los horizontes de subida del nivel medio del mar para el año 2100 en cada mareógrafo fueron los siguientes:

1. Cálculo de la tendencia de cambio del nivel medio del mar ( $\mathrm{mm} /$ año) en cada mareógrafo de la costa española mediante regresión lineal, en el periodo más largo disponible para cada uno de los mareógrafos (Penland y Ramsey, 1990). Aunque frecuentemente se citan otras aproximaciones para este tipo de cálculos como la descomposición en periodos armónicos para aislar el término de la pendiente (Pugh, 1987; Tel y García, 2004; Marcos et al., 2005), estas exigen de una continuidad de las series temporales de los mareógrafos y de una densidad de la red de mareógrafos que no se ajustan a los datos disponibles para todas las costas peninsulares.

2. Cálculo de la tendencia de cambio del nivel medio en la serie global publicada por el CSIRO (Church y White, 2012) en el periodo de coincidencia con cada uno de los mareógrafos estudiados, mediante regresión lineal. Dado que es frecuente encontrar lagunas temporales en las series anuales de los ma- 
reógrafos, se reelaboró una serie del CSIRO para cada mareógrafo, que incluyera las mismas lagunas, año de inicio y año de finalización que el mareógrafo de referencia. De este modo, se garantizó que la comparación de las tendencias globales y locales se realizara sobre series idénticas en su dimensión temporal.

3. Cálculo de la diferencia entre la tendencia de cambio del nivel medio del mar en el mareógrafo y en la serie del CSIRO. Se estimó esta diferencia como el «factor local», considerado como ajeno a eventos globales de subida del nivel medio del mar tanto pasados como futuros (Titus y Narayanan, 1998).

4. Estimación del coeficiente de Correlación de Pearson entre las series de registros locales y la serie global. Se descartaron las series temporales con correlaciones no significativas de acuerdo con el test de la t de Student para $=0,1$ (Ebdon, 1984).

5. Elección de un escenario y modelo de cambio global del nivel medio del mar. Para el presente trabajo se emplearon los modelos que aparecen en la tabla 2.

TABLA 2

MODELOS, ESCENARIOS Y SUBIDAS ESPERADAS DEL NIVEL MEDIO DEL MAR AL FINAL DEL SIGLO XXI

\begin{tabular}{lcc}
\hline \multicolumn{1}{c}{ MODELO } & ESCENARIO & SUBIDA $(\mathrm{m})$ \\
\hline IPCC (2013) & RCP2.6 & 0,40 \\
IPCC (2013) & RCP4.5 & 0,47 \\
IPCC (2013) & RCP8.5 & 0,63 \\
Rahmstorf (2006) & - & 0,90 \\
Pfeffer (2008) & Low 1 & 0,80 \\
Pfeffer (2008) & High 1 & 2,00 \\
\hline
\end{tabular}

6. Cálculo de la subida local del nivel medio del mar en 110 años (periodo 1990 / 2100), a partir de la ecuación 1, para los modelos de Rahmstorf y Pfeffer.

$$
\operatorname{SLR}=(L * 110)+G
$$


Siendo:

SLR Subida local del nivel medio del mar en el año 2100.

L Factor local identificado en la comparación de niveles medios del mar registrados localmente y globalmente.

$G$ El valor de la subida global identificado por el modelo de cambio del nivel del mar empleado.

Para los modelos procedentes del informe de 2013 del IPCC se empleó una ecuación semejante a la 1, multiplicando por 96 años en lugar de por 110, ya que la subida del nivel medio del mar se calcula en relación al período que va del promedio de 1986/2005 al promedio de 2081/2100 (ecuación 2).

$$
S L R=(L * 96)+G
$$

\section{RESULTADOS}

El primer grupo de resultados obtenidos aparecen sintetizados en las tablas 3 y 4 , y consisten en las tendencias de cambio del nivel del mar resultantes del análisis de las series temporales de cada mareógrafo y de la serie del CSIRO reelaborada para cada mareógrafo, y en las diferencias observadas entre ambas. De las series temporales mayores de 30 años (que cumplen la longitud mínima señalada por la OMM para series temporales de datos climáticos (Cuadrat y Pita, 1996) e incluso superiores a 50-60 años (Tel, 2007), las mayores diferencias observadas entre la tendencia local de cada mareógrafo y la global se encuentran en el mareógrafo de Cádiz, con una diferencia de $+2.01 \mathrm{~mm} /$ año.

El resto de mareógrafos con series largas del nivel medio del mar se mantienen en valores relativamente bajos, con factores locales cercanos a 0 (Málaga, A Coruña, Vigo, Santander), con la excepción del mareógrafo de Alicante, cuyos resultados quedan invalidados para el propósito de este estudio por haberse identificado un coeficiente de correlación de Pearson negativo.

Las diferencias entre las tendencias globales y locales registradas en los mareógrafos de series más cortas (que comienzan a medir desde la década de los 90 hasta el presente) muestran una mayor variabilidad, con valores que oscilan entre $+3,8 \mathrm{~mm}$ /año de diferencia (Barcelona) y -2,19 mm /año (Bilbao). Las series temporales de los mareógrafos de Almería fueron descartadas por presentar valores del coeficiente de correlación de Pearson por debajo del umbral de significación elegido. 
TABLA 3

TENDENCIAS DE CAMBIO DEL NIVEL DEL MAR EN LOS MAREÓGRAFOS ESPAÑOLES PENINSULARES CON SERIES DE MÁS DE 30 AÑOS, EN LAS SERIES

DEL CSIRO ADAPTADAS, DIFERENCIAS Y CORRELACIONES ENTRE AMBAS

\begin{tabular}{lcccccc}
\hline \multicolumn{1}{c}{ Mareógrafo } & $\begin{array}{c}\text { Periodo } \\
\text { observado }\end{array}$ & Años & $\begin{array}{c}\text { Tendencia } \\
\text { de la serie } \\
\text { (mm/año) }\end{array}$ & $\begin{array}{c}\text { Tendencia global } \\
\text { observada en ese } \\
\text { periodo (mm/año) }\end{array}$ & $\begin{array}{c}\text { Diferencia } \\
\text { global/local }\end{array}$ & Correlación \\
\hline CÁDIZ & $1960-2011$ & 47 & 3,95 & 1,94 & 2,01 & 0,70 \\
ALGECIRAS & $1944-2011$ & 43 & 0,40 & 1,82 & $-1,42$ & 0,25 \\
MÁLAGA1 & $1962-2011$ & 45 & 2,34 & 1,96 & 0,38 & 0,25 \\
ALICANTE2 & $1960-1995$ & 34 & $-0,80$ & 1,25 & $-2,05$ & $-0,54$ \\
SANTANDER1 & $1944-2012$ & 51 & 2,15 & 1,82 & 0,33 & 0,69 \\
A CORUÑA1 & $1944-2012$ & 58 & 2,39 & 1,81 & 0,58 & 0,73 \\
VIGO & $1943-2012$ & 59 & 2,29 & 1,81 & 0,48 & 0,78 \\
\hline
\end{tabular}

TABLA 4

TENDENCIAS DE CAMBIO DEL NIVEL DEL MAR EN LOS MAREÓGRAFOS ESPAÑOLES PENINSULARES DE CORTA DURACIÓN, EN LAS SERIES DEL CSIRO ADAPTADAS, DIFERENCIAS Y CORRELACIONES ENTRE AMBAS

\begin{tabular}{lcccccc}
\hline \multicolumn{1}{c}{ Mareógrafo } & $\begin{array}{c}\text { Periodo } \\
\text { observado }\end{array}$ & Años & $\begin{array}{c}\text { Tendencia } \\
\text { de la serie } \\
(\mathrm{mm} / \text { año) }\end{array}$ & $\begin{array}{c}\text { Tendencia global } \\
\text { observada en ese } \\
\text { periodo (mm/año) }\end{array}$ & $\begin{array}{c}\text { Diferencia } \\
\text { global/local }\end{array}$ & Correlación \\
\hline HUELVA & $1996-2011$ & 13 & 5,63 & 3,18 & 2,45 & 0,708 \\
BONANZA & $1992-2011$ & 17 & 5,69 & 3,18 & 2,51 & 0,822 \\
MÁLAGA2 & $1992-2011$ & 17 & 4,78 & 2,85 & 1,93 & 0,832 \\
ALMERÍA1 & $1978-1997$ & 18 & 0,29 & 1,52 & $-1,23$ & $-0,240$ \\
VALENCIA & $1993-2011$ & 16 & 6,03 & 2,84 & 3,18 & 0,642 \\
BARCELONA & $1993-2011$ & 14 & 6,61 & 2,78 & 3,83 & 0,847 \\
BILBAO & $1993-2011$ & 15 & 0,65 & 2,84 & $-2,19$ & 0,604 \\
GIJÓN2 & $1995-2011$ & 14 & 1,88 & 2,26 & $-0,38$ & 0,860 \\
\hline
\end{tabular}


Los resultados obtenidos del cálculo de las expectativas de subida del nivel medio del mar en cada uno de los mareógrafos analizados aparecen en las tablas 5 y 6 . En ellos se observa una amplia variabilidad, tanto entre diferentes localizaciones como dentro de cada mareógrafo al modificar los modelos de cambio global.

\section{TABLA 5}

SUBIDAS ESPERADAS (M) DEL NIVEL MEDIO DEL MAR AL FINAL DEL SIGLO XXI EN LOS MAREÓGRAFOS ESPAÑOLES PENINSULARES DE SERIES LARGAS PARA DIFERENTES ESCENARIOS DE CAMBIO GLOBAL

\begin{tabular}{lcccccc}
\hline \multicolumn{1}{c}{ Mareógrafo } & RCP2.0 & RCP4.5 & RCP8.0 & PfefferLow 1 & Pfeffer High 2 & Rahmstorf \\
\hline CÁDIZ & 0,59 & 0,66 & 0,81 & 1,02 & 2,22 & 1,12 \\
ALGECIRAS & 0,26 & 0,33 & 0,48 & 0,64 & 1,84 & 0,74 \\
MÁLAGA1 & 0,44 & 0,51 & 0,66 & 0,84 & 2,04 & 0,94 \\
SANTANDER1 & 0,43 & 0,50 & 0,65 & 0,84 & 2,04 & 0,94 \\
A CORUÑA1 & 0,46 & 0,53 & 0,68 & 0,86 & 2,06 & 0,96 \\
VIGO & 0,45 & 0,52 & 0,67 & 0,85 & 2,05 & 0,95 \\
\hline
\end{tabular}

TABLA 6

SUBIDAS ESPERADAS DEL NIVEL MEDIO DEL MAR (M) AL FINAL DEL SIGLO XXI EN LOS MAREÓGRAFOS ESPAÑOLES PENINSULARES DE SERIES CORTAS PARA DIFERENTES ESCENARIOS DE CAMBIO GLOBAL

\begin{tabular}{lcccccc}
\hline \multicolumn{1}{c}{ Mareógrafo } & RCP2.0 & RCP4.5 & RCP8.0 & PfefferLow 1 & Pfeffer High 2 & Rahmstorf \\
\hline HUELVA & 0,64 & 0,71 & 0,86 & 1,07 & 2,27 & 1,17 \\
BONANZA & 0,64 & 0,71 & 0,86 & 1,08 & 2,28 & 1,18 \\
MÁLAGA2 & 0,59 & 0,66 & 0,81 & 1,01 & 2,21 & 1,11 \\
VALENCIA & 0,71 & 0,78 & 0,93 & 1,15 & 2,35 & 1,25 \\
BARCELONA & 0,77 & 0,84 & 0,99 & 1,22 & 2,42 & 1,32 \\
BILBAO & 0,19 & 0,26 & 0,41 & 0,56 & 1,76 & 0,66 \\
GIJÓN2 & 0,36 & 0,43 & 0,58 & 0,76 & 1,96 & 0,86 \\
\hline
\end{tabular}


En los mareógrafos de la costa andaluza (figura 5) se observa una menor variabilidad que en el resto de la Península, coherente con unas diferencias entre las subidas globales y locales menos pronunciadas. Aun así, la magnitud esperada de la subida del nivel medio del mar es muy alta en los mareógrafos de Cádiz y de Málaga, con valores que oscilan entre 0.50 y $1 \mathrm{~m}$ de acuerdo al modelo del IPCC y los 3 escenarios elegidos, y entre 0.90 y $2.25 \mathrm{~m}$ de acuerdo con los modelos de Rahmstorf y Pfeffer. Sin embargo, en el mareógrafo de Algeciras, que presenta una menor tendencia de cambio del nivel medio del mar (próxima a $0 \mathrm{~mm} /$ año), los valores esperados de subida se encuentran entre 0.26 y $0.48 \mathrm{~m}$ para los escenarios del IPCC y 0.64 y $1.84 \mathrm{~m}$ para los modelos empíricos críticos con el IPCC. Los mareógrafos de Huelva y Bonanza (con series temporales sensiblemente más cortas), mostraron expectativas de cambio del nivel medio del mar ligeramente superiores a las de los mareógrafos de Cádiz y Málaga, de un máximo de 0.20 m más.

\section{FIGURA 5}

RESULTADOS OBTENIDOS PARA LOS MAREÓGRAFOS LOCALIZADOS EN LAS COSTAS ANDALUZAS

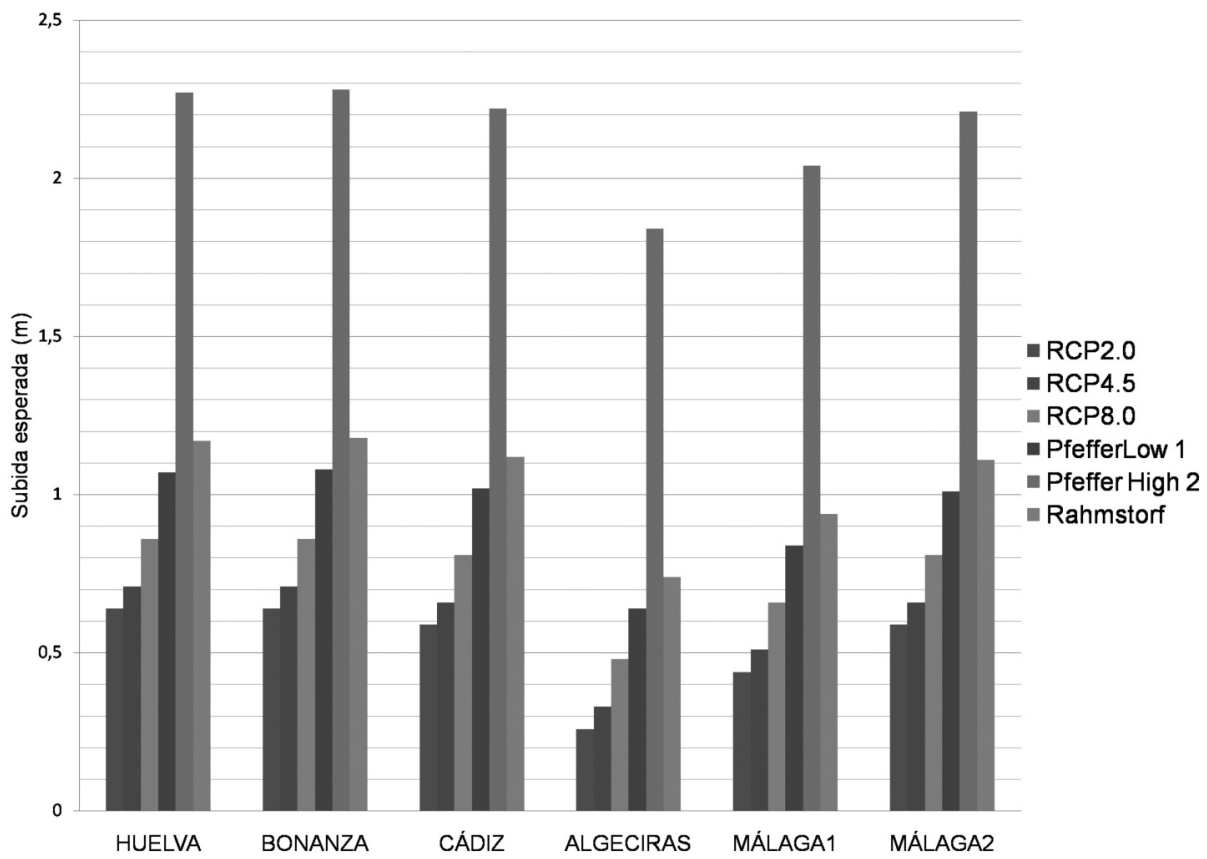

Estudios Geográficos, Vol. LXXVII, 280, pp. 57-79, enero-junio 2016 ISSN: 0014-1496, eISSN: 1988-8546, doi: 10.3989/estgeogr.201603 
Los mareógrafos de la costa gallega y cantábrica muestran valores muy homogéneos (figura 6), oscilando las expectativas de los escenarios del IPCC en la horquilla de 0.35-0.85, con la excepción de Bilbao, cuya tendencia de cambio del nivel medio del mar excepcionalmente baja en el contexto nacional muestra valores entre 0.04-0.19 para el caso de los escenarios del IPCC, y que incluso en el modelo de Pfeffer, que en casi todos los mareógrafos superan los 2.5 metros, apenas llega a 2.09 metros.

FIGURA 6

\section{RESULTADOS OBTENIDOS PARA LOS MAREÓGRAFOS LOCALIZADOS EN LAS COSTAS CANTÁBRICAS Y DE GALICIA}

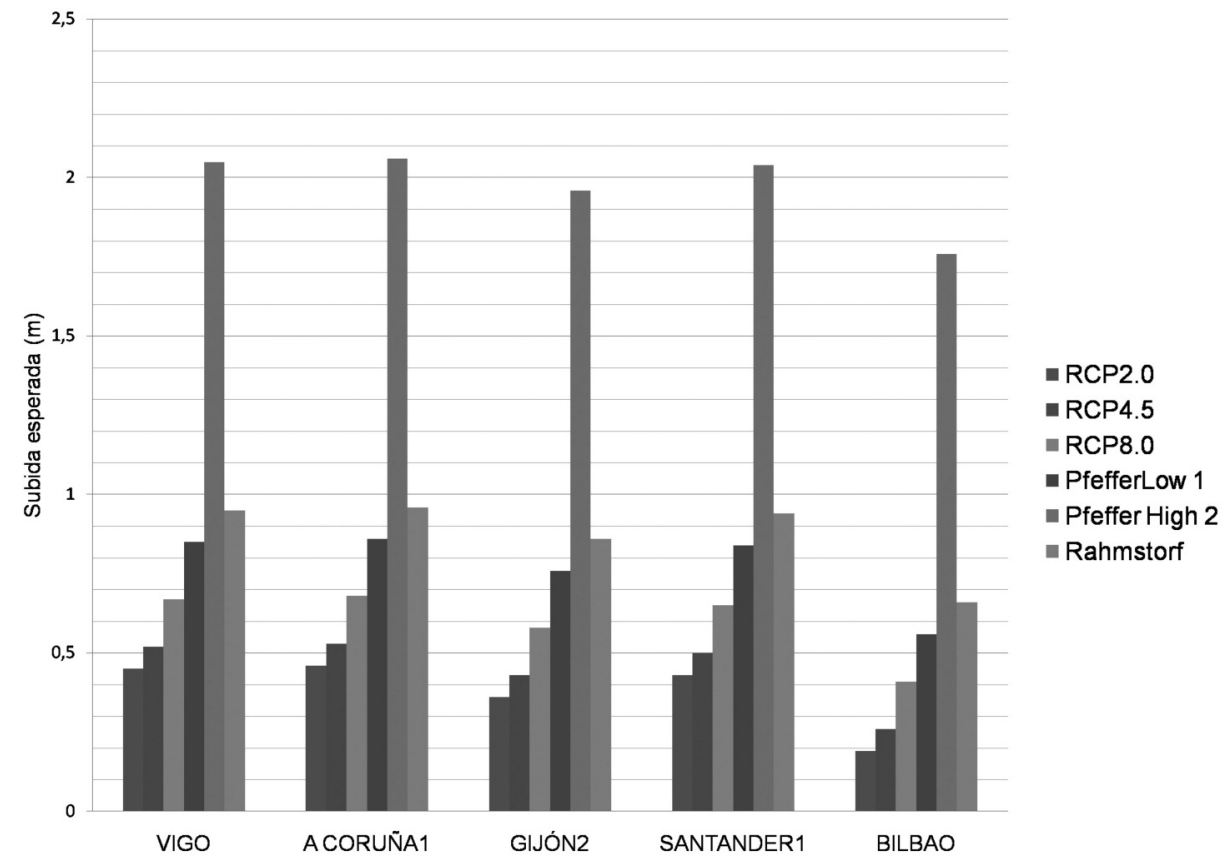

La costa mediterránea (figura 7) destaca por la escasa longitud de las series de sus mareógrafos (solo el de Alicante presenta registros de más de 30 años, y ha tenido que ser eliminado del estudio por presentar una correlación negativa con la serie global), y la similitud de valores esperados entre los mareógrafos de Barcelona y Valencia. 
FIGURA 7

RESULTADOS OBTENIDOS PARA LOS MAREÓGRAFOS LOCALIZADOS EN LAS COSTAS VALENCIANA Y CATALANA

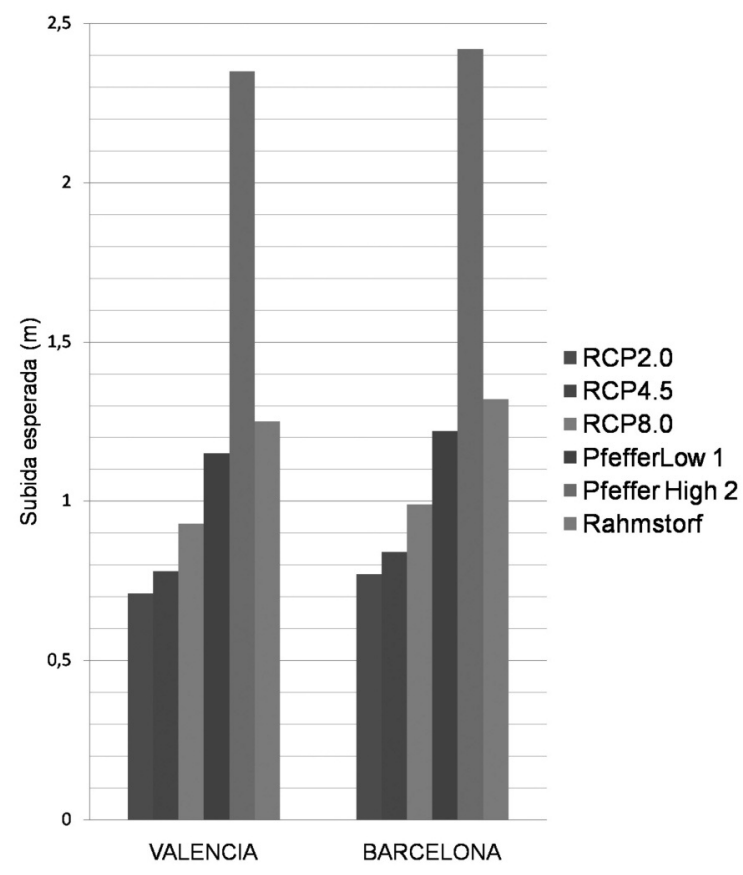

\section{DisCUSIÓN}

De las tres variables a partir de las cuales se han calculado las predicciones de subida del nivel medio del mar (modelo matemático de cambio global, escenario de emisiones futuro, y tendencias locales observadas), los resultados obtenidos muestran una gran dependencia del modelo matemático elegido, por encima de los diferentes escenarios futuros y de las tendencias locales. Estas diferencias se acentúan en el caso del escenario «alto» (High) del modelo de Pfeffer, del cual se derivan expectativas de subidas del nivel medio del mar que frecuentemente duplican los resultados obtenidos a partir del modelo del IPCC, en cualquiera de sus escenarios.

Por ello, la resolución del debate científico planteado en torno a la cuestión de la magnitud de la subida futura del nivel del mar por partes del IPCC y autores críticos con sus asunciones resultará esencial para poder realizar predic- 
ciones de mayor precisión. La magnitud de la variabilidad causada por la elección de uno u otro modelo se sitúa muy por encima del rango de variación observado entre los escenarios del IPCC, cuyas relativamente pequeñas desigualdades se deben, sin embargo, a grandes diferencias en las expectativas de comportamiento de la sociedad futura en cuanto a emisión de gases durante el resto del siglo XXI.

En cualquier caso, el modelo de Pfeffer presenta un comportamiento muy diferente del IPCC, al obtener resultados muy diferentes en función de si se emplea el escenario alto o bajo. De hecho, prescindiendo del escenario alto de Pfeffer, las tendencias locales observadas se muestran como la variable de mayor importancia en el análisis de expectativas futuras de subida del nivel del mar. Estas diferencias se acentúan en los mareógrafos con series temporales cortas. Es muy probable que este fenómeno se explique por la fuerte dependencia de los valores iniciales y finales en el cálculo de tendencias obtenidas a partir de análisis de regresión lineal en series temporales, lo cual obliga a tomar con precauciones los resultados obtenidos a partir de este tipo de series.

Los resultados obtenidos en el análisis de las tendencias pasadas de cambio del nivel medio del mar resultan muy semejantes a los publicados con anterioridad tanto por Tel y García (2004) con diferentes métodos de análisis para la mayor parte de los mareógrafos con series largas de registros como por Moreno (2008), lo cual parece indicar una menor relevancia del método de análisis frente a la calidad de la serie temporal y sobre todo del modelo global de cambio del nivel medio del mar elegido para estimar expectativas de cambio en el futuro.

De este modo, cobra una singular relevancia la discusión existentes entre los modelos «conservadores» propuestos por el IPCC (2007 y 2013) y los modelos semiempíricos basados en la constatación de las diferencias entre las predicciones realizadas en el pasado y las observaciones (Rahmstorf, 2007; Rahmstorf, 2010; Horton et al., 2008; Pfeffer et al., 2008; Bitterman y Rahmstorff, 2014). La confrontación de ambos tipos de modelos no resulta concluyente ni en último informe del IPCC (2013) ni en trabajos independientes de esta institución (Grinsted et al., 2014; Nicholls y Cazenve, 2010), por lo que la discusión de los resultados obtenidos en este trabajo obliga a considerar todos los escenarios posibles.

Las implicaciones de la elección de uno u otro modelo para los costas españolas son semejantes tanto en trabajos semejantes realizados en áreas insulares (Fraile et al., 2014; López Torres et al., 2015) como en las peninsulares, de acuerdo con los resultados obtenidos en este trabajo, así como la evidente dependencia del escenario elegido. 
Metodológicamente, este trabajo incorpora la comparación entre los registros locales y los globales para poder estimar el comportamiento futuro del nivel del mar, frente a la más común asunción de un único umbral de carácter global para todo el planeta (Nichols y Cazenave, 2010; Curtis y Schneider, 2011). Aunque es un concepto desarrollado inicialmente por Titus y Narayanan (1998), la novedad de la eliminación en la serie global de los registros perdidos en las series locales permite una comparación homogénea entre datos locales y globales. Esto permite adaptar este tipo de aproximaciones metodológicas a la red de mareógrafos españoles, singularmente pobre por el elevado número de lagunas en las series temporales en comparación con las series de mareógrafos comúnmente empleadas en otros estados.

El cálculo de la correlación observada entre la serie global y la serie local resulta un indicador de la fiabilidad de la predicción realizada, en el caso de que se cumplan las asunciones que llevan a la ocurrencia de uno $u$ otro escenario de cambio global. En este sentido, los autores desean subrayar que no se trata de un indicador sobre la calidad de la serie del mareógrafo, sino que ha de ser interpretado exclusivamente como un indicador de la semejanza en la variabilidad del nivel del mar a escala local con respecto a la global. No obstante, es probable que en algunos casos sobre los que se han reportado dudas acerca de la fiabilidad de las series temporales (Alicante, Gibraltar), los bajos valores de correlación obtenidos que llevaron a descartar los resultados para estos mareógrafos, podrían estar explicados por inhomogeneidades estadísticas presentes en las series temporales de los mareógrafos. Tal y como ha sido reportado (Ebdon, 1985), los valores del coeficiente de correlación de Pearson han de interpretarse considerando la longitud de las series temporales. Por ello, la semejanza en los valores obtenidos para este coeficiente entre los mareógrafos con series largas (Cádiz, Santanter, A Coruña y Vigo) y los mareógrafos con series cortas (Huelva, Bonanza, Málaga. Valencia, Barcelona, Gijón) otorgan más fiabilidad al primer grupo que al segundo.

Los resultados obtenidos evidencian importantes diferencias entre mareógrafos, tanto en las tasas de cambio del nivel registradas como en las expectativas de cambio futuro, dependientes de estas últimas. Aunque las diferencias entre observaciones locales y globales han sido interpretadas comúnmente desde el punto de vista de la isostasia (Peltier, 1999), e incluso ha sido incorporada en algunos análisis realizados en España (Tel y García, 2004) esta aproximación no permite explicar las fuertes diferencias encontradas en mareógrafos situados a idénticas latitudes en incluso de gran proximidad. En este sentido, la explicación de este tipo de diferencias se puede explicar desde tres posibles causas: i) la existencia de cambios locales absolutos en la superficie 
marina, sobre los que recientemente se ha subrayado su alta variabilidad espacial (Woodworth y Menéndez, 2015), ii) la presencia de cambios relativos explicados por fenómenos de tectónica local como fallas o compactación de áreas sedimentarias, que pueden llegar a afectar a las propias infraestructuras sobre las que se asientan los mareógrafos (Pugh, 2004), y iii) errores en las series temporales de los mareógrafos, identificadas en trabajos anteriores (Fraile, 2011), y que en el caso de los errores más evidentes han obligado a descartar fragmentos de las series analizadas (como en algunas series que comienzan a finales del siglo XIX o durante los años 40 del siglo Xx, de dudosa fiabilidad) en este mismo trabajo, sin que ello permita descartar otros posibles errores no detectados.

Espacialmente, la pobreza de la red de mareógrafos españoles (en este sentido, no muy diferente de las del resto de estados europeos, por ejemplo) impide interpretar los resultados en términos regionales, puesto que la densidad de mareógrafos es mínima en relación al tamaño de las principales unidades de relieve. Los datos obtenidos son coherentes con trabajos de cambios absolutos registrados en la superficie marina (Cazenave et al., 2001), aunque cualquier diferencia observada en mareógrafos puede ser interpretada por causa de los movimientos verticales de la superficie emergida (Wöppelmann y Marcos, 2012). Los resultados obtenidos resultan asimismo coherentes con otros análisis realizados mediante serie de mareógrafos en condiciones similares en otros territorios (Pirazzoli, 1987; Carillo et al., 2014).

\section{CONCLUSIONES}

El método propuesto permite estimar subidas futuras a partir de series de niveles del mar de longitud inferior a 100 años, lo cual supone un considerable avance sobre otros trabajos basados en la propuesta de Titus y Narayanan (1998) sobre la estabilidad de los fenómenos locales en la evolución temporal de las series del nivel medio del mar. En cualquier caso, al mostrar los mareógrafos con series temporales cortas (inferiores a 30 años) una mayor variabilidad en sus resultados aquellos con series largas, es evidente que el método desarrollado resulta más certero cuanto mayor sea la longitud de la serie del mareógrafo, tal y como es común a cualquier análisis temporal. Estimar la correlación entre las series temporales analizadas y la serie global de referencia es un requisito imprescindible para poder realizar predicciones con cierta fiabilidad.

La mayor importancia del factor local frente a las variables escenario y modelo (con la salvedad del escenario alto de Pfeffer), recalca la necesidad de re- 
alizar este tipo de estudios de carácter local, y permite descartar el empleo de valores únicos procedentes de modelos globales para cualquier ámbito de la costa. No obstante, este hecho pone de relevancia que el análisis presentado en este trabajo tan solo abarca ámbitos puntuales, quedando aun amplios sectores costeros entre cada mareógrafo sobre los que no hay información fidedigna. Las técnicas de interpolación espacial y análisis de los registros altimétricos deberán soslayar en el futuro estas carencias.

En todos los mareógrafos de la costa española se esperan subidas del nivel medio del mar para el año 2100, independientemente del comportamiento local de los cambios relativos del nivel del mar y del modelo y escenario empleado para el análisis. Incluso las menores subidas obtenidas de entre todos los resultados obtenidos resultan lo suficientemente amplias como para esperar que se produzcan cambios relevantes en aspectos ecológicos, económicos (erosión costera) y para los asentamientos humanos (intensificación de los procesos de inundación episódica asociadas a temporales, o inundaciones permanentes provocadas por las pleamares), lo cual debería llevar a considerar la subida del nivel medio del mar, con su variabilidad espacial, dentro del ámbito de la planificación territorial.

\section{REFERENCIAS}

Bittermann, K. y Rahmstorf, S. (2014): "Using proxy data to calibrate a semi-empirical sea-level model", en R. Gert-Jan (ed.), EGU General Assembly Conference Abstracts, 16, Viena, European Geosciences Union, p. 12.673.

Bosello, F., Nicholls, R. J., Richards, J., Roson, R. y Tol, R. S. (2012): "Economic impacts of climate change in Europe: sea-level rise", Climatic change, 112/1, pp. 63-81.

Bruce, J. P., Hoesung, L. y Haites, E. F. (eds.) (1996): Climate change 1995: Economic and social dimensions of climate change: Contribution of Working Group III to the second assessment report of the Intergovernmental Panel on Climate Change, Cambridge, University Press, $448 \mathrm{pp}$.

Carillo, A., Sannino, G., Artale, V., Ruti, P. M., Calmanti, S. y Dell'Aquila, A. (2012): "Steric sea level rise over the Mediterranean Sea: present climate and scenario simulations", Climate dynamics, 39/9-10, pp. 2.167-2.184.

Cazenave, A., Cabanes, C., Dominh, K. y Mangiarotti, S. (2001): "Recent sea level change in the Mediterranean Sea revealed by Topex/Poseidon satellite altimetry", Geophysical research letters, 28/8, pp. 1.607-1.610.

Church, J. A. y White, N. J. (2011): "Sea-level rise from the late 19th to the early 21st century", Surveys in Geophysics, 32/4-5, pp. 585-602. 
Cuadrat J. M. y Pita M. F. (1996): Climatología, Madrid, Cátedra, 496 pp.

Curtis, K. J. y Schneider, A. (2011): "Understanding the demographic implications of climate change: estimates of localized population predictions under future scenarios of sea-level rise", Population and Environment, 33/1, pp. 28-54.

Ebdon, D. (1985): Statistics in geography, Oxford, Basil Blackwell, 242 pp.

Emrich, C. T. y Cutter, S. L. (2011): "Social vulnerability to climate-sensitive hazards in the southern United States", Weather, Climate, and Society, 3/3, pp. 193-208.

Fraile Jurado, P. (2011): Análisis de las problemáticas asociadas a la espacialización, evolución y representación de niveles del mar presentes y futuros en Andalucía, Sevilla, Universidad de Sevilla, 362 pp.

Fraile Jurado, P. y Ojeda Zújar, J. (2012): "Evaluación de la peligrosidad asociada al aumento de la superficie inundable por la subida del nivel medio del mar en la costa entre Cádiz y Tarifa”, GeoFocus. Revista Internacional de Ciencia y Tecnología de la Información Geográfica, 12, pp. 329-348.

Fraile-Jurado P. y Ojeda-Zújar, J. (2013): "The importance of the vertical accuracy of digital elevation models in gauging inundation by sea level rise along the Valdelagrana beach and marshes (Bay of Cádiz, SW Spain)", Geo-Marine Letters, 33, pp. 225-230.

Fraile Jurado, P., Sánchez Rodríguez, E., Fernández Díaz, M., Pita López, M. F. y López Torres, J. M. (2015): "Estimación del comportamiento futuro del nivel del mar en las Islas Canarias a partir del análisis de registros recientes", Geographicalia, 66, pp. 79-98.

Grinsted, A., Jevrejeva A y Riva, R. (2014): "Sea level rise projection for Northern Europe", en R. Gert-Jan (ed.), EGU General Assembly Conference Abstracts, 16, p. 1.614.

Hallegatte, S., Ranger, N., Mestre, O., Dumas, P., Corfee-Morlot, J., Herweijer, C. y Wood, R. M. (2011): "Assessing climate change impacts, sea level rise and storm surge risk in port cities: a case study on Copenhagen", Climatic change, 104/1, pp. 113-137.

Horton, R., Herweijer, C., Rosenzweig, C., Liu, J., Gornitz, V. y Ruane, A. C. (2008): "Sea level rise projections for current generation CGCMs based on the semiempirical method", Geophysical Research Letters, 35/2, pp. 1-5.

Intergovernmental Panel on Climate Change (IPCC) (2001): Climate Change 2001: The Scientific Basis, New York, Cambridge University Press, 94 pp.

Intergovernmental Pannel on Climate Change (IPCC) (2007): Cambio climático 2007, Impactos, adaptación y vulnerabilidad, New York, Cambridge University Press, 41 pp.

Internation Panel on Climate Change (IPCC) (2013): Climate Change 2013: The Physical Science Basis. Contribution of Working Group I to the Fifth Assessment Report of the Intergovernmental Panel on Climate Change, New York, Cambridge University Press, $1.535 \mathrm{pp}$.

Karl, T. R., Melillo, J. M. y Peterson, T. C. (2009): Global climate change impacts in the United States, New York, Cambridge University Press, 196 pp. 
Katsman, C. A., Sterl, A., Beersma, J. J., Van den Brink, H. W., Church, J. A., Hazeleger, W. y Weisse, R. (2011): "Exploring high-end scenarios for local sea level rise to develop flood protection strategies for a low-lying delta- Sea level rise projections for current generation CGCMs based on the semiempirical method. The Netherlands as an example", Climatic change, 109/3-4, pp. 617-645.

Lizitsin, E. (1974): Sea-level changes, Amsterdam, Elsevier Sci., 285 pp.

Loáiciga, H. A., Pingel, T. J. y Garcia, E. S., (2012): "Sea Water Intrusion by Sea Level Rise: Scenarios for the 21st Century", Ground water, 50/1, pp. 37-47.

López Torres, J. M., Fraile Jurado, P. y Sánchez Rodríguez, E. (2015): “Cartografía de inundaciones por subida del nivel del mar en las islas orientales de Canarias", Geo-temas, 15, pp. 201-204.

Marcos, M., Gomis, D., Monserrat, S., Alvarez-Fanjul, E., Pérez, B. y García-Lafuente, J. (2005): "Consistency of long sea-level time series in the northern coast of Spain", Journal of Geophysical Research, 3, p. 110.

Méndez, F. J., Medina, R., Losada, I. J., Olabarrieta, M., Tomás, A., Liste, M. y Castanedo, S. (2004): "Estudio de los impactos en la costa española por efecto del cambio climático", en J. C. García Codrón y A. Hernández Gimena (eds.), El Clima entre el Mar y la Montaña, Santander, Asociación Española de Climatología y Universidad de Cantabria, pp. 64-66.

Moreno, J. M. (2005): Principales conclusiones de la evaluación preliminar de los impactos en España por efecto del cambio climático, Madrid, Secretaría General de la Contaminación y del Cambio Climático, 40 pp.

Moreno, J. M. (2006): "Evaluación preliminar de los impactos en España por efecto del cambio climático", Boletín CF+ S, 38, pp. 38-39.

Moss, R. H. (2011): "Reducing doubt about uncertainty: Guidance for IPCC's third assessment", Climatic change, 108/4, pp. 641-658.

Nicholls, R. J. (2011): "Planning for the impacts of sea level rise", Oceanography Society, 24/2, p. 144.

Nicholls, R. J. y Cazenave A. (2010): "Sea-level rise and its impact on coastal zones", Science, 328/5985, 1517-1520.

Nicholls, R. J., Hanson, S. E., Lowe, J. A., Warrick, R. A., Lu, X., Long, A. J. y Carter, T. R. (2011): Constructing sea-level scenarios for impact and adaptation assessment of coastal areas: A guidance document, Ginebra, Intergovernmental Panel on Climate Change Task Group on Data and Scenario Support for Impacts and Climate Analysis, pp. 1-47.

Pardo Pascual, J. E. (1989): "Oscillacions del nivell del mar a partir de dades mareogràfiques", Cuadernos de Geografía, 46, pp. 107-126.

Peltier, W. R. (1999): "Global sea level rise and glacial isostatic adjustment", Global and Planetary Change, 20/2, 93-123.

Penland, S. y Ramsey, K. E. (1990): "Relative sea-level rise in Louisiana and the Gulf of Mexico: 1908-1988", Journal of Coastal Research, 1, pp. 323-342.

Pfeffer, W. T., Harper, J. T. y O'Neel, S. (2008): "Kinematic constraints on glacier contributions to 21st-century sea-level rise”, Science, 321/5894, pp. 1340-1343. 
Pirazzoli, P. A. (1987): "Sea level changes in the Mediterranean", en M. J. Tooley \& I. Shennan (eds.), Sea level changes, London, Institut of British Geographers, pp. 152-181.

Pugh, D. (2004): Changing sea levels: effects of tides, weather and climate, Cambridge, Cambridge University Press, 263 pp.

Pugh, D. T. (1987): Tides, Surges and Mean Sea-Level: A Handbook for Engineers and Scientists, Swingdon, Reino Unido, John Wiley \& Sons Ltd., 472 pp.

Rahmstorf, S. (2007): "A semi-empirical approach to projecting future sea-level rise", Science, 315/5810, pp. 368-370.

Rahmstorf, S. (2010): "A new view on sea level rise", Nature reports climate change, 4, pp. 44-45.

Schubert, R., Schellnhuber, H. J., Buchmann, N., Epiney, E., Griebhammer, R., Kulessa, M. y Schmid, J. (2006): The future oceans-Warming up, rising high, turning sour, Berlín, Wissenschaftlicher Beirat Globale Umweltveränderungen, 123 pp.

Tel, E. (2007): Variabilidad y tendencias del nivel del mar en las costas de la Península Ibérica y zonas limítrofes: su relación con parámetros meteorológicos, Madrid, Instituto Español de Oceanografía, 349 pp.

Tel, E. y García, M. J. (2004): "Nivel del mar en las costas españolas y su relación con el clima”, en J. C. García Codrón, y A. Hernández Gimena, (eds.), El Clima entre el Mar y la Montaña, Santander, Asociación Española de Climatología y Universidad de Cantabria, pp. 135-144.

Titus, J. G. y Anderson, K. E. (2009): Coastal sensitivity to sea level rise: a focus on the mid Atlantic Region, Washington, Government Printing Office, 320 pp.

Titus, J. G. y Narayanan, V. K. (1998): The Probability of Sea Level Rise, Washington, DIANE Publishing, 196 pp.

Wodworth, P. L. y Menéndez, M. (2015): "Changes in the mesoscale variability and in extreme sea levels over two decades as observed by satellite alimetry", Journal of Geophysical Research: Oceans, 210/1, pp. 64-77.

Wöppelmann, G. y Marcos, M. (2012): "Coastal sea level rise in southern Europe and the nonclimate contribution of vertical land motion", Journal of Geophysical Research: Oceans, 117, pp. 1978-2012.

Fecha de recepción: 30 de enero de 2014.

Fecha de aceptación: 28 de octubre de 2015.

\section{RESUMEN}

En este trabajo se realiza una estimación de las expectativas de cambio del nivel medio del mar en el año 2100 en los mareógrafos peninsulares de España. Mediante el análisis temporal de las tendencias de cambio anual observadas en los mareógrafos y su relación con el comportamiento global del nivel medio del mar durante el periodo de medida de cada mareógrafo, se calculó un factor local que permite modificar, al 
alza o a la baja, las expectativas globales de cambio enunciadas por diferentes modelos globales de cambio del nivel medio del mar en el futuro, obteniendo una previsión local. Los cálculos se realizaron para 18 mareógrafos y 6 escenarios diferentes de subida del nivel del mar. Los resultados obtenidos muestran subidas generalizadas en todos los casos, aunque con importantes diferencias, que dependen tanto del escenario elegido para el análisis como del comportamiento pasado del nivel del mar en cada mareógrafo.

Palabras Clave: subida del nivel del mar; mareógrafo; pronóstico; serie temporal; escenario.

\section{AsbTract}

The aim of this work is to calculate mean sea level rise expectatives for the year 2100 in the tide gauges located in the Spanish side of the Iberian Peninsula. By means of the use of time series analysis of annual mean sea level change rates, and comparing these data with the evolution of the global mean sea level during the period of measurement of each tide gauge, a local factor of sea level rise was obtained. The local factor is used to modify the future global expectatives, obtaining a local future value. The calculations have been made for 18 tide gauges and 6 different scenarios of mean sea level rise. The obtained results show generalized sea level rises, with strong differences depending on the global sea level rise scenario and on the recent evolution of mean sea level in each tide gauge.

KEY WORDS: mean sea level rise; tide gauge; future expectatives; time series; scenario. 